RESEARCH REPORT

\title{
ESTIMATION OF ODDS RATIO AMONG CERVICAL RADICULOPATHY PATIENTS WITH MIGRAINE
}

\begin{abstract}
OBJECTIVES

To estimate the probability of migraine among cervical radiculopathy patients by using odds ratio.

\section{STUDY SETTINGS \& PARTICIPANTS}

This study was carried out at two tertiary care hospitals of Karachi, Out-Patient Department (OPD) of Rehabilitation Sciences, Ziauddin Hospital North and Liaquat National Hospital.

\section{METHOD}

Cross-sectional study was performed on participants aged between 25 to 45 years with both diagnosed and undiagnosed cervical radiculopathy to estimate the risk of migraine in comparison to healthy individuals. The characteristics features of migraine were assessed on migraine assessment scale while odds ratio was calculated to determine the odds.
\end{abstract}

\section{RESULTS}

The patients with $\mathrm{CR}$ are at higher risk for developing migraine as compared to the healthy individuals (odds ratio 1.2, 95\% confidence interval.

\section{CONCLUSIONS}

The study concluded that patients cervical radiculopathy have increased risk of migraine. Therefore, evaluation of the condition in cervical radiculopathy patients for assessing the susceptibility for migraine is crucial for the effective management of migraine.

\section{KEY WORDS}

Radiculopathy, Migraine Disorder, Odds Ratio, Spondylosis, Migraine, Cervicogenic Headache.

\author{
Najma Zehra \\ Physiotherapist \\ Dr. Ziauddin Hospital \\ Dia.zahra@gmail.com
}

[Zehra N. A Estimation of Odds Ratio among Cervical Radiculopathy Patients With Migraine. Pak. j. rehabil.2019;8(2):32-37] 


\section{INTRODUCTION}

Cervical radiculopathy is an age-related disorder which occurs due to compression of cervical nerve roots. A population-based analysis reported an annual incidence of cervical radiculopathy of 107.3 per 100,000 for men and 63.5 per 100,000 for women with a peak at 50 to 54 years of age'. Due to age-related degenerative changes, spinal wear and tear increases which cause neck pain and disability'. In young individuals, a sudden injury could cause cervical radiculopathy that untimely leads to a herniated disk. According to annual reports about the incidence of $C R$, the incidence rate of this disease is 83.2 cases per 100,000 persons and its prevalence rate is 3.5 cases per 1000 people. In most of the cases of cervical radiculopathy, C6/C7 or C5/C6 motion segments are damaged ${ }^{2}$. The patients with CR complaint suffers from severe neck pain which radiated to arm and hand due to which sensory and motor functions of arm and hand are disturbed ${ }^{3}$. Migraine imparts considerable effects on the patient's quality of life. It imposes a significant socioeconomic burden as well ${ }^{4}$ Patients with cervical radiculopathy complain of neck pain and radiating pain in the arm sometimes combined with sensory and motor disturbances in the arm and/or hand $^{4}$. These symptoms are accepted as being caused by the nerve root compression. Headache, if asked for, is frequently reported by patients with cervical radiculopathy but in general not discussed in terms of nerve root compression in the lower cervical spine. On the other hand, affection of nerve structures in the upper cervical spine, including the C2 and C3 nerve roots and the occipital major nerve, are accepted as a cause of occipital headache ${ }^{4-5}$. The term cervicogenic headache, is often mentioned in the literature. It is not related to nerve root compression, but described as being caused by musculoskeletal structures in the upper cervical spine and the pathogenic mechanism is unclear5.The criteria for cervicogenic headache are recurrent and strictly unilateral headache without side shift. It starts in the neck and spreads to the fronto-ocular area and is elicited by certain movements or awkward position of the head. Clinical investigation reveals reduced mobility of the neck and external pressure tenderness is present ${ }^{5}$. Studies suggested that headache in patients with cervical radiculopathy is more disabling for the patient than the radicular pain in the shoulder/arm. Many hypotheses have ascertained that there is a causal relationship between migraine and its triggering factors ${ }^{5-6}$. Among these triggers, cervical pathologies may initiate the sequence of events that results in migraine symptoms. The extensive functional convergence of upper cervical spinal cord from the descending fibers in the trigeminal nucleus caudalis, which terminates within the trigeminocervical nucleus, and the afferent fibers from the upper cervical roots, which communicate in this region, accounting for the bi-directional pathway of pain between the neck and head. This interaction refers the cervical pathologies to the head, which is the activity also proposed to cause cervicogenic headache' . Constantly noxious cervical afferent irritation via this pathway is a possible key element in causing migraines.Moreover, cervicogenic headache is caused by the cervical pathologies to the head that leads to constant harmful irritation of cervical afferent which is the most important factor for causing migraine. According to the musculoskeletal anatomy, muscle tension and neck pain could be consequences of neck injuries ${ }^{6}$ Neck pain and cervical muscle tenderness are common and prominent symptoms of primary headache disorders. Less commonly, head pain may actually arise from bony structures or soft tissues of the neck, a condition known as cervicogenic headache. Cervicogenic headache can be a perplexing pain disorder that is refractory to treatment if it is not recognized ${ }^{5-6}$. The condition's pathophysiology and source of pain have been debated, but the pain is likely referred from one or more muscular, neurogenic, osseous, articular, or vascular structures in the neck. Moreover muscle tension is another common migraine symptoms and both could be sequelae of neck injuries, according to the musculoskeletal anato$\mathrm{my}^{6}$. Moreover, administering multiple injections in targeted head and neck regions is sometimes considered important for the management of migraines, cervicogenic headaches, and myofascial referred pain ${ }^{6}{ }^{7}$ indicating that headache and neck pain may share some common pathways. Although a previous report indicated that cervical spondylosis (CS) accounts for $15.9 \%$ of migraineurs ${ }^{7}$, until now epidemiological evidence of a link between CS and the risk of migraine is minimal. Therefore, we conducted this nationwide retrospective cohort study to investigate the longitudinal causal relationship between CS and migraines and CS severity in relation to the risk of developing a migraine?.

For many years, headaches related to neck grabbed the attention of many researchers. According to Pearce, headaches related to neck disorders were being diagnosed since $1860^{7}$. Previous studies have shown that middle-aged and young females with cervical radiculopathy were highly susceptible to develop migraine as compared to other individuals ${ }^{8}$. The MRI examination of these patients verified that due to degenerative diseases their cervical spines were damaged at one or more levels of lower cervical spine. Moreover, it was also reported that patients were able to distinguish headache from migraine?. Regarding the classification of headache, it may be difficult to distinguish migraine without aura, tension type headache and cervicogenic headache in patients with neck pain ${ }^{10}$. The researchers performed a retrospective cohort study from 2000 to 2011 in which 27,930 patients with cervical spondylosis participat- 
ed in the study. For evaluating the relationship between cervical spondylosis and migraine, the researchers applied multivariate and univariate proportional hazard regression analysis. The findings of the study ascertained that patients with cervical spondylosis were highly susceptible to migraine as compared to those without cervical spondylosis. Furthermore, the incidence rate of migraine was also higher in patients' with cervical spondylosis ${ }^{11}$. It was also reported that headache disorders such as migraine and cervical musculoskeletal abnormalities had bi-directional comorbidity. The pain clinics reported migraine in $25 \%$ of patients with cervicogenic headache and two-third patients with neck pain'2. Likewise, Riina et al (2009) determined the postoperative effects of headaches in patients with cervical radiculopathy. The findings revealed that about $90 \%$ of patients with cervical radiculopathy had headaches including migraine. Similar findings were consistent with the previous studies that predicted the incidence of migraine in CR patients $^{13}$, however the findings were not generalized with respect to different population therefore more evidence needs to be established in this regard.

\section{METHODOLOGY}

A cross-sectional study was performed on participants aged between 25 to 45 years with both diagnosed and undiagnosed cervical radiculopathy to estimate the risk of migraine in comparison to healthy individuals. The characteristics features of migraine were assessed on migraine assessment scale while odds ratio was calculated to determine the odds.

\section{Inclusion Criteria}

Both male and female patients aged between 25 to 45 years with diagnosed cervical radiculopathy of C4-T1. Normal individuals without cervical radiculopathy with similar age group.

\section{Exclusion Criteria}

Individuals with vertebral fractures, congenital vertebral anomalies, surgical interventions and pregnancy.

\section{Data Collection Tool}

The self-designed questionnaire consists of enquiries to assess migraine in the participants with diagnosed radiculopathy and normal individuals. The questions were related to the severity and duration of pain, if the pain is severe and for more than 72 hours which was triggered by light, stress or weather change and patient also feels nauseated then it was considered highly suggestive of migraine. Other questions were helpful to evaluate either the headache was due to migraine or not. Further the probability of migraine was calculated by the Odd Ratio.

\section{Data Collection Procedure}

Data was collected through a self-designed questionnaire following their informed consent that was taken to maintain the ethical consideration of confidentiality. The questionnaire was used to asses' migraine in the participants based on the inclusion criteria. Moreover, the questionnaire contained the demographic information, gender and occupation questions are related to the severity of pain, pattern of headache, factors that trigger their headache and its effect on their activities of daily living. The total score of the questionnaire was 21 i.e. the individuals scoring a maximum of 10 score were considered as positive sign of migraine.

\section{Data Analysis Procedure}

Data was entered and analyzed on SPSS (Statistical Package for Social Sciences) version 20. Participants demographic details were demonstrated through descriptive statistics consists of frequency, mean and standard deviations whereas ratio of migraine in exposed and unexposed population with cervical radiculopathy will be calculated by Odds Ratio.

\section{RESULTS}

A total number of 50 participants were included in this study (20 male and 30 females) comprised of 27 , 19 and 9 cases of healthy, patients with cervical radiculopathy and spasm respectively 30.3 years. Furthermore, participants were belonged to different occupations/professions or other work as shown in Table-1.

\begin{tabular}{|l|l|}
\hline \multicolumn{2}{|l|}{ Table.1 Demographic Characteristics } \\
\hline Participants & $(\mathrm{n}=50)$ \\
\hline Males & 20 \\
\hline Females & 30 \\
\hline Age in Years Mean (SD) & $\begin{array}{l}30.3 \\
(4.6)\end{array}$ \\
\hline Occupation & \multicolumn{2}{|l|}{} \\
\hline Doctors & 3 \\
\hline Physical Therapist & 14 \\
\hline Occupational Therapist & 2 \\
\hline Office Workers & 17 \\
\hline Others & 14 \\
\hline Musculoskeletal Disorder & 19 \\
\hline Cervical Radiculopathy & 4 \\
\hline Cervical Spasm & 27 \\
\hline Normal & \multicolumn{2}{|l}{} \\
\hline
\end{tabular}


On Migraine Assessment Scale, it was found that migraine severity varied among individuals from mild (16\%), moderate (52\%) and severe (32\%) with least duration of $<1$ hour in $16 \%$ while more than $2-4$ hours in $46 \%$ patients as illustrated in Figure- 1. Furthermore, $64 \%$ participants reported trigging of headache due to stress, change of weather or chocolates. Although, medicine $(54 \%)$ and rest $(34 \%)$ was found to be effective in relieving pain as shown in Table-2.

\begin{tabular}{|c|c|}
\hline \multicolumn{2}{|c|}{ Table.2 Migraine Assessment Scale } \\
\hline Headache Referred to Neck & $35(70 \%)$ \\
\hline \multicolumn{2}{|l|}{ Severity of Pain } \\
\hline Mild & $8(16 \%)$ \\
\hline Moderate & $26(52 \%)$ \\
\hline Severe & $16(32 \%)$ \\
\hline Nausea in Headache & $25(50 \%)$ \\
\hline $\begin{array}{l}\text { Effect of Light during } \\
\text { Headache }\end{array}$ & $21(42 \%)$ \\
\hline $\begin{array}{l}\text { Triggering of Headache } \\
\text { due to Stress, Weather } \\
\text { Change and Chocolates }\end{array}$ & $32(64 \%)$ \\
\hline $\begin{array}{l}\text { Effect of Headache on } \\
\text { Speech }\end{array}$ & $6(12 \%)$ \\
\hline $\begin{array}{l}\text { Effect of Headache on } \\
\text { Physical Activity }\end{array}$ & $28(56 \%)$ \\
\hline \multicolumn{2}{|l|}{ Duration of Headache } \\
\hline Less Than 1 Hour & $8(16 \%)$ \\
\hline 1 Hour & 7 (14\%) \\
\hline 2 to 4 Hours & $23(46 \%)$ \\
\hline More Than 4 Hours & $12(245)$ \\
\hline Migraine in Family & $6(12 \%)$ \\
\hline \multicolumn{2}{|l|}{ Relieve in Headache } \\
\hline Medicine & 54 \\
\hline Exercise & 6 \\
\hline Rest & 34 \\
\hline Massage & 6 \\
\hline
\end{tabular}

\begin{tabular}{|l|c|c|c|}
\hline \multicolumn{4}{|c|}{ Table.3 Risk Estimation } \\
\hline & Value & \multicolumn{2}{|c|}{$95 \% \mathrm{Cl}$} \\
\hline $\begin{array}{l}\text { Odds Ratio of } \\
\text { Migraine } \\
\text { (Cervical } \\
\text { Radiculopathy } \\
\text { /Healthy) }\end{array}$ & 1.2 & Upper & Lower \\
\cline { 2 - 4 } & & 0.9 & 0.4 \\
\hline
\end{tabular}

The Odds Ratio of risk estimation for migraine is 1.2.Therefore, an odds ratio of 1.2 with a confidence interval of 0.4 to 0.9 suggested that there is a $95 \%$ probability that odds ratio would lie in the range of 0.4 to 0.9 if there are no confounding or biases as shown in Table-3.

\section{DISCUSSION}

This study determined the risk of estimation of migraine in patients with cervical radiculopathy. The pathophysiological mechanisms underlying many of the classifications of headache are not well understood ${ }^{4}$. In terms of research evaluation, migraine has received the most attention, and it is believed to involve abnormal brain function, but the pathophysiology is still not clearly defined ${ }^{15} \mathrm{CGH}$ arises primarily from musculoskeletal dysfunction in the upper three cervical segments ${ }^{16}$. The pathway by which pain originating in the neck can be referred to the head is the trigemino cervical nucleus which descends in the spinal cord to the level of $\mathrm{C} 3 / 4$, and is in anatomical and functional continuity with the dorsal gray columns of these spinal segments ${ }^{17}$ Hence, input via sensory afferents principally from any of the upper three cervical nerve roots may mistakenly be perceived as pain in the head a concept known as convergence ${ }^{18}$. The findings ascertain the hypothesis examined in this study that patients with cervical radiculopathy are highly susceptible to develop migraine as compared to healthy individuals. Headache disorders and musculoskeletal abnormalities of head and neck were bi-directional comorbidities. According to previous studies, about $90 \%$ of symptoms with cervical radiculopathy or myelopathy had anterior cervical surgery for relieving headache ${ }^{14}$. Moreover, studies reported that patients with headache disorders presented areas of muscle tenderness, postural changes and the myofascial trigger points when they were compared with healthy individuals or individual without headache ${ }^{15}$. Cervical musculoskeletal abnormalities and headache disorders were a bi-directional comorbidity, with nearly two-thirds of migraineurs coexisting with neck pain or stiffness, and one-fifth of patients with cervicogenic headache in pain clinics ${ }^{15}$. Previous studies have disclosed that almost $90 \%$ patients with headache accepted anterior cervical operation for the treatment of symptoms with cervical myelopathy or radiculopathy ${ }^{15}$. Furthermore, the authors bserved that patients with headache disorders presented the myofascial trigger points, clear-cut areas of muscle tenderness, and posture changes, when compared with non-headache control ${ }^{14}{ }^{15}$. Thus linking between cervical spine disorder and migraine, emphasizing the importance of assessment for migraine at first and follow-up period in CS patients due to the increasing risk for the development of migraine. Previous studies have shown that middle-aged and young females with cervical radiculopathy were highly susceptible to develop migraine as compared to other individuals ${ }^{16}$ On the other hand, cervical radiculopathy affects verte- 
brae and discs of the spinal cord that is an age-related disorder ${ }^{17}$. In addition, the neuroplastic changes in the brain and spinal cord produced by persistent peripheral nociceptive impulses lead to pain and central sensitization. Thus, the development of migraine requires a combination of central disinhibition and of tonic nociceptive input 18. Therefore, it has been suggested to ascertain the severity of radiculopathy with the help of advanced imaging ${ }^{19}$. According to Allison and Ditor, the actual mechanism cause pathogenesis of cervical spondylotic myelopathy that leads to the migraine attack is the activation of the inflammatory cascade. The excessive release of the pro-inflammatory molecules into the bloodstream stimulates the hypothalamic-pituitary-adrenal axis that leads to hypothalamic dysfunction ${ }^{20}$. According to previous studies, as a result of the degenerative processes of spinal cords vertebrae in the neck, the capsular ligaments of facet joints is damaged which leads to the hypermobility of upper cervical vertebrae ${ }^{21}$. Further, this dysregulation of the vertebrobasilar arteries results from such cervical instability which produces migraine headache ${ }^{22}$. Besides, the symptoms of migraine could be reproduced when myofascial trigger points are stimulated ${ }^{23}$. The prolonged and recurrent irritated inputs associated with pain could worsen and provoke migraine attacks $^{24}$. There are several clinical implications of the study in public health. The physicians must assess the patient with cervical radiculopathy for migraine as early as possible because minimizing the risk for developing migraine disorder ${ }^{25}$. Moreover, our findings demonstrated that incidence rate of migraine was higher among the patients with cervical radiculopathy as compared to the healthy individuals, which suggest that further studies could be conducted on evaluating the influence of cervical radiculopathy severity for determining the severity of migraine headache.

\section{CONCLUSION}

The study concluded that patients cervical radiculopathy have increased risk of migraine. Therefore, evaluation of the condition in cervical radiculopathy patients for assessing the susceptibility for migraine is crucial for the effective management of migraine. Moreover, further studies are needed to analyze the associated risk factor related to cervical radiculopathy for the estimation of risk among patients that is potential to predict patient's prognosis.

\section{REFERENCES}

[1] Lyer, S., \& Kim, H. J. Cervical radiculopathy. Current reviews in musculoskeletal medicine, 2016. 9(3), 272-280.

[2] Shabat S, Leitner J, Folman Y. Pulsed radiofrequency for the suprascapular nerve for patients with chronic headache. J Neurosurg
Anesthesio. $2013 ; 25(3): 340-3$.

[3] Choi BW, Kim SS, Lee DH, Kim JW. Cervical radiculopathy combined with cervical myelopathy: prevalence and characteristicsEuropean Bone and Joint Infection Society. $2017 ; 27(7): 889-93$.

[4] Lin, W. S., Huang, T. F., Chuang, T. Y., Lin, C. L., \& KaO, C. H. 2018. Association between cervical spondylosis and migraine: a nationwide retrospective cohort study. Int. j. environ. res, 15(4), 587.

[5] Martin PR, Reece J, Callan M, MacLeod C, Kaur A, Gregg K, Goadsby PJ. Behavioral management of the triggers of recurrent headache: a randomized controlled trial. Behaviour research and therapy. 2014;61:1-1.

[6] Biondi D, Bajwa Z. Cervicogenic headaches. Up To date [Online].[cited 2013.

[7] Sinaki M, Lee M, Garza I. Successful management of cervicogenic headaches in kyphotic posture through mechanical and spinal proprioceptive intervention program: A case series. J Med Therap. 2018;2:4-5.

[8] Vetvik, K.G. and MacGregor, E.A., 2017. Sex differences in the epidemiology, clinical features, and pathophysiology of migraine. The Lancet Neurology, 16(1), pp.76-87

[9] Puledda F, Messina R, Goadsby PJ. An update on migraine: current understanding and future directions. Neurology. 2017 264(9):2031-9.

[10] Schrot RJ, Mathew JS, Li Y, Beckett L, Bae HW, Kim KD. Headache relief after anterior cervical discectomy: post hoc analysis of a randomized investigational device exemption trial. Journal of Neurosurgery: Spine. 2014;21 (2):217-22.

[1 1] Liang Z, Galea O, Thomas L, Jull G, Treleaven J. Cervical musculoskeletal impairments in migraine and tension type headache: A systematic review and meta-analysis. Musculoskeletal Science and Practice. 2019.

[12] Lin, W.S., Huang, T.F., Chuang, T.Y., Lin, C.L. and Kao, C.H., 2018. Association between cervical spondylosis and migraine: a nationwide retrospective cohort study. International Int J Environ Res Public Health, 15(4), p.587.

Cleveland C. The Effects of Manual Therapy

[13] and Therapeutic Exercise on a Young Patient with Chronic Cervicogenic Headaches: A Case Study.

[14] Schrot, R.J., Mathew, J.S., Li, Y., Beckett, L., Bae, H.W. and Kim, K.D., 2014. Headache relief after anterior cervical discectomy: post hoc analysis of a randomized investigational device exemption trial. Journal of Neurosurgery: Spine, 21 (2), pp.217-222.

[15] Janani AS, Pope KJ, Fenton N, Grummett TS, Bakhshayesh $H$, Lewis TW, Watson DH, Whitham EM, Willoughby JO. Resting cranial and upper cervical muscle activity is increased in patients with migraine. Clinical Neurophysiology. $2018 ; 129(9): 1913-9$. 
[16] Vetvik, K.G. and MacGregor, E.A., 2017. Sex differences in the epidemiology, clinical features, and pathophysiology of migraine. The Lancet Neurology, 16(1), pp.76-87.

[17] Wang, C., Tian, F., Zhou, Y., He, W. and Cai, Z., 2016. The incidence of cervical spondylosis decreases with aging in the elderly, and increases with aging in the young and adult population: a hospital-based clinical analysis. Clinical interventions in aging, 1 1, p.47.

[18] Vardeh D, Mannion RJ, Woolf CJ. Toward a mechanism-based approach to pain diagnosis J Pain2016;17(9):T50-69.

[19] Murphy, R.K., Sun, P., Xu, J., Wang, Y., Sullivan, S., Gamble, P., Wagner, J., Wright, N.N., Dorward, I.G., Riew, D. and Santiago, P., 2016. Magnetic resonance imaging biomarker of axon loss reflects cervical spondylotic myelopathy severity. Spine, 41 (9), p.751.

[20] Allison, D.J. and Ditor, D.S., 2015. Immune dysfunction and chronic inflammation following spinal cord injury. Spinal cord, 53(1), p.14.

[21] Steilen, D., Hauser, R., Woldin, B. and Sawyer,
S., 2014. Chronic neck pain: making the connection between capsular ligament laxity and cervical instability. Open Orthop J, 8, p.326.

[22] Shum GL, Cinnamond S, Hough AD, Craven R, Whittingham W. Test-Retest Reliability of Measuring the Vertebral Arterial Blood Flow Velocity in People With Cervicogenic Dizziness. J Manipulative Physiol Ther. 2017;40(4):255-62.

[23] Menezes AH. Craniovertebral junction abnormalities with hindbrain herniation and syringomyelia: regression of syringomyelia after removal of ventral craniovertebral junction compression. J Neurosurg. $2012 ; 116(2): 301-9$.

[24] Fernández-De-Las-Peñas, C. and Cuadrado, M.L., 2014. Therapeutic options for cervicogenic headache. Expert review of neurotherapeutics, 14(1), pp.39-

[25] Slatculescu AM, Chen Y. Synergism between female gender and high levels of daily stress associated with migraine headaches in Ontario, Canada. Neuroepidemiology. 2018;51 (3-4): 183-9. 